\title{
Immobilization of drugs for glaucoma treatment
}

\author{
Jose Filipe Almeida - Anabela Fonseca • \\ Cristina M. S. G. Baptista · Eugénio Leite · \\ Maria Helena Gil
}

Received: 15 November 2005/ Accepted: 21 August 2006/Published online: 14 June 2007

(C) Springer Science+Business Media, LLC 2007

\begin{abstract}
Recently there have been some developments in the preparation of controlled drug delivery systems for glaucoma. Many materials are being used in this area, namely gelatine and chitosan. Both of them present high levels of biocompatibility and biodegradability. In this paper, we wish to report the work we have been doing on the preparation and characterization of hydrogels based on gelatine and chitosan. The crosslinking agents used were 1(3-(Dimethylamino)propyl)-3-Ethylcarbodiimide hydrocholide (CDI), 1,4-Butanodiol diglycidyl ether (epoxyde 1), Ethylene glycol diglycidyl ether (epoxyde 2) and genipin. The results obtained showed that all of the films were hydrogels. The surface and transversal cut showed a porous surface in all the films. The thermal analysis proved the modifications in the polymeric chains, with the stabilization of all of them by the crosslinking agents. The release pattern indicates that the gelatine films were the best since they release the adequate proportion of drug. Finally, the cytotoxicity showed that the gelatine films were all biocompatible, specially the ones crosslinked with one of the Epoxydes.
\end{abstract}

\section{Introduction}

Glaucoma is one of the diseases that affect the internal human eye. It is due to an increase of the intraocular

J. F. Almeida · A. Fonseca · C. M. S. G. Baptista

E. Leite · M. H. Gil ( $₫)$

Departamento de Engenharia Química, Universidade de Coimbra, Polo II, Rua Sílvio Lima, Coimbra 3030-790, Portugal

e-mail: hgil@eq.uc.pt pressure (IOP) which results from the production and drainage of the aqueous humour. In its worst case it can lead to total loss of vision by the patient [1].

The most common type of glaucoma is the primary open angle that can be treated by topical administration of drugs, namely beta-blockers (such as Timolol, Betaxolol or Latanoprost), associated with another type of drugs to control the IOP, such as Pilocarpina [2, 3]. Due to the working mechanism of the eye, the quantity of drugs that really reaches the local of activity is very low (normally 5\%) [3]. So, to maintain the concentrations of the drug, it is necessary that the patient make many applications during the day. To avoid this situation, drug delivery systems have been developed. These systems are characterized by the ability of releasing the right proportion of drug during long periods of time. Besides the drug can be delivered in the right place in the body [4].

The liberation can occur in many ways, namely due to controlled diffusion of the drug through the film porous, or due to the degradation of the polymeric matrix [5].

When the release occurs in accordance with the diffusion process of the drug, the diffusion parameters can be calculated. For this purpose, Fick's 1st law has to be applied [6]:

$J_{A}=-D_{A, B} \frac{d C_{A}}{d x}$,

where, $J_{A}$ represents the molar flux of the substance $A$, express in $\mathrm{mg} /\left(\mathrm{m}^{2} \mathrm{~s}\right), D_{A, B}$ is the diffusion coefficient of $A$ in $B$, express in $\mathrm{m}^{2} / \mathrm{s}$ and $d C_{A} / d x$ is the gradient of concentrations of substance A on a specific axel. In this case study, after the beginning of the release, the concentrations of the drug in both sides of the film change. So the gradient is not constant and, in the limit, it 
will reach zero. Therefore in accordance with the second Fick's Law:

$\frac{d C_{A}}{d t}=-D_{A, B} \frac{d^{2} C_{A}}{d x^{2}}$

Rearranging the expression, the value of the coefficient of diffusion can be calculated:

$\frac{M_{t}}{M_{\infty}}=\frac{4}{\pi^{\frac{1}{2}}} \sqrt{\frac{D t}{l_{0}^{2}}}$,

where $M_{\infty}$ is the total amount of drug used in the preparation of the system, $M_{t}$ the amount of drug released in a time $t$, and $l_{0}$ is half the film's thickness. This expression is valid for $M_{t} / M_{\infty}<0.5$.

Several polymers, e.g. PVA, PVP, chitosan and gelatine [7-9], have been used in the development of drug delivery systems.

These polymers can be considered as hydrogels, solutions of hydrophilic polymers, with great capacity of water absorption $[10,11]$. In order to crosslink the above polymers, various crosslinking agents have been used, like polyepoxydes [12], Genipin [13, 14], N-N'-Carbonilodiimidazol or Carbodiimide [15, 16].

The present paper describes new ways to modify both chitosan and gelatine, in order to prepare new films to be used as drug delivery systems. The films were characterized in different ways, namely, their swelling rate, and their thermal transitions. The surface and the transversal cuts were also characterized by SEM. Finally, we present the results for the drug delivery kinetics and the cytotoxicity of the gelatine membranes.

\section{Materials and methods}

Materials

Gelatine from porcine skin (EC 232-554-6) was purchased from Sigma (Steinheim, Germany). Chitosan, (medium molecular weight), 1-(3-(Dimethylamino)propyl)-3-Ethylcarbodiimide hydrocholide, 98+\% (CDI), 1,4-Butanodiol diglycidyl ether, tech., 60\% (EC 219-371-7) and Ethylene glycol diglycidyl ether, tech. 50\% (EC 218-746-2) were purchase from Aldrich Chemical Company (Steinheim, Germany). Genipin, a natural crosslinking agent, was acquired from Challenge Bioprodutcs Co., Ltd.

Acetic Acid, with a degree of purity of $99.5 \%$, was purchase from BHD-Chemicals Lda (Poole, UK), and ethanol to dissolve 1,4-Butanodiol diglycidyl ether, was acquire from Pronolab (Lisbon, Portugal).
The drug used during this study was Timolol Maleate obtain from Edol (Portugal).

Preparation of the gelatine solution

In order to prepare the gelatine solution, $2 \mathrm{~g}$ of gelatine powder were dissolved in $10 \mathrm{~mL}$ of distillate water at $50{ }^{\circ} \mathrm{C}$, with mechanical stirring for $20 \mathrm{~min}$.

Preparation of the chitosan solution

In order to prepare a $1.33 \%$ solution, $2 \mathrm{~g}$ of chitosan were dissolved in $150 \mathrm{~mL}$ of an acetic acid solution at $5 \%(\mathrm{w} / \mathrm{v})$, for $24 \mathrm{~h}$ at room temperature.

Crosslinking of gelatine

Several gelatine films, with different crosslinking agents, were prepared. Their composition is indicated in Table 1.

Film 1 was prepared without any crosslinking agent. This film was considered as a blank test in all the experiments. Membranes 2, 3 and 4 were prepared by adding, to the $10 \mathrm{~mL}$ of the gelatine solution, the CDI, previously dissolved in a minimal quantity of distillate water to allow a better dispersion of the crosslinking agent in all the polymeric solution.

For the membranes crosslinked with Ethylene glycol diglycidyl ether (epoxyde 2), the procedure was much easier, because the compound is in the liquid state, which allows a better dispersion without the needing of a solubilization in water.

Finally, the third crosslinking agent used was genipin, a natural compound that was added directly to the solution.

Crosslinking of chitosan

The chitosan, in solution, was crosslinked by using two different crosslinking agents, 1,4-Butanodiol diglycidyl

Table 1 Percentage of three crosslinking agents used in the preparation of gelatine based films

\begin{tabular}{lll}
\hline Film number & Crosslinking agent & Concentration (W/W) $(\%)$ \\
\hline 1 & - & - \\
2 & CDI & 1 \\
3 & CDI & 2 \\
4 & CDI & 3 \\
5 & Epoxyde & 0.2 \\
6 & Epoxyde & 0.3 \\
7 & Epoxyde & 1 \\
8 & Genipin & 1 \\
9 & Genipin & 2 \\
\hline
\end{tabular}


Table 2 Crosslinking agents and their concentrations used in the chitosan films

\begin{tabular}{lll}
\hline Film number & Crosslinking agent & Concentration $(\mathrm{W} / \mathrm{W})(\%)$ \\
\hline 10 & Epoxyde 1 & 1 \\
11 & Epoxyde 1 & 2 \\
12 & Epoxyde 2 & 0.2 \\
13 & Epoxyde 2 & 0.5 \\
14 & Epoxyde 2 & 1 \\
15 & - & - \\
\hline
\end{tabular}

ether (epoxyde 1) and Ethylene glycol diglycidyl ether (epoxyde 2). The concentrations used are presented in Table 2.

In the preparation of the chitosan films, $10 \mathrm{~mL}$ of the solution previously made were used. Due to the hidrophobicity of epoxyde 1, it was dissolved in an ethanol solution before the mixing with the chitosan solution under mechanical stirring. As for epoxyde 2, the method used was equal to the one used for gelatine. Finally, the last film was made to serve as a blank test.

Before the introduction of the crosslinking agent, we put, as for the gelatine films, $5 \mathrm{mg}$ of drug previously dissolved in water.

In both cases, the drug was immobilized by entrapment, i.e., it was dissolved in the solutions before addition of the crosslinking agents.

After the formation of the final solution, we put them on a petri dish and left to dry to open air, at $20{ }^{\circ} \mathrm{C}$ during $72 \mathrm{~h}$.

Physical characterisation of the membranes

\section{Swelling ratio determination}

All the films, with weights between 200 and $400 \mathrm{mg}$ were introduced into a beaker containing $400 \mathrm{~mL}$ of physiological serum at $18{ }^{\circ} \mathrm{C}$ and kept, until constant weight was achieved. Then the films were clean with filler paper and weighed every minute for a period of $15 \mathrm{~min}$. From the graph obtained, the initial weight was evaluated for each experiment. The swelling capacity was determined by using the following equation:

$\%$ swelling $=\frac{M_{\text {wet }}-M_{\text {dry }}}{M_{\text {dry }}} \times 100$

where $M_{\text {wet }}$ is the weight of the films after reaching constant weight in serum and $M_{\text {dry }}$ the weight of each films completely dry.
Morphological analysis (SEM)

The preparation of the samples to SEM was made in three different steps. First, we removed the water of the samples by liophilization in vacuum, at temperatures near $-50{ }^{\circ} \mathrm{C}$.

After $48 \mathrm{~h}$, the samples were put on a metallic support and covered with gold in order to make the material a good conductor. Finally, each sample was analysed by SEM on a JSM-5310.

\section{Thermal Analysis studies (DSC)}

The tests of Thermal Analysis were made in a PL-DSC, with a constant flux of nitrogen of $3-4 \mathrm{~mL} / \mathrm{s}$, at a $1,2 \mathrm{bar}$ pressure. The results were analysed with help of a computer program call Thermal Analysis.

The samples for each test had a weight between 5 and $6 \mathrm{mg}$ and were subject to a heating process from 30 to $200{ }^{\circ} \mathrm{C}$, at a constant velocity of $10^{\circ} \mathrm{C} / \mathrm{min}$. All the samples were analysed in duplicate.

\section{Study of the releasing kinetic of drugs from the polymeric} films

In this part of the study, the quantification of the amount of drug released was made with the use of the Ultraviolet absorption technique with help of a spectrophotometer Jasco V-530. The films were introduced in a dialysis membrane to avoid, during the degradation process, any piece of the film interfering in the reading. Then all films were put in Erlemeyers containing $100 \mathrm{~mL}$ of physiological serum. Each assay was carried out at $37^{\circ} \mathrm{C}$. The absorption readings were made after $15 \mathrm{~min}, 30 \mathrm{~min}, 1,2$, $3, \ldots, 24,25, \ldots, 196 \mathrm{~h}$ at $294 \mathrm{~nm}$ of wavelength.

\section{Biocompatibility and cytotoxicity tests}

After the preparation of the films, they were washed with physiological serum during $24 \mathrm{~h}$, in order to remove any trace of the crosslinking agent that didn't react. The weight of each film was between 10 and $60 \mathrm{mg}$, and each sample was made in duplicate. The tests were made with the films hydrated in order to avoid any absorption of the medium by the samples. After this wash, they were sterilized with UV radiations, in a P-Selecta UV Estéril, at $254 \mathrm{~nm}$ during $40 \mathrm{~min}$.

In order to analyse the cytotoxicity of each film, the SRA 01/04 cells (Epithelial Cells of Lens) and the COS-7 (isolated cells from the suprarenal African Green Monkey) were chosen. The cells were grown in DMEM (Dulbecco's Modified Eagle Medium) with glucose and glutamine ad- 
ded with $10 \%$ of bovine fetal serum and antibiotic estuptomicin/penicillin.

The cells were grown at $37{ }^{\circ} \mathrm{C}$ in a carbon dioxide and atmosphere and $100 \%$ of humidity. The medium was changed every two days. The cell density in the holes was $0.1 \times 10^{6}$ cells $/ \mathrm{mL}$.

For each plate we made a blank test, only with cells and cell medium. The viability study, with the films in contact with the cells, was held during $24 \mathrm{~h}$, and the test chosen was the MTT (M-5655 Thiazolyl Blue). $40 \mu \mathrm{L}$ of a MTT solution of $5 \mathrm{mg} / \mathrm{mL}$ were added, prepared in PBS (Phosfate Buffer Solution) in each hole of the plate. The plate was then incubated for $2 \mathrm{~h}$ during which the MTT was metabolised by the living cells into an insoluble formazan compound. The crystals obtained were dissolved in $300 \mathrm{~mL}$ of isopropanol acid. From that volume, we took $150 \mathrm{~mL}$ to put in the Multiwell Plates with 96 holes and made an Elisa reading in the spectrophotometer at $450 \mathrm{~nm}$.

\section{Results and discussion}

In the first crosslinking reaction, $\mathrm{CDI}$ activates $-\mathrm{COOH}$ groups from different aminoacids that lately will crosslink with $-\mathrm{NH} 2$ groups of other aminoacids.

Figures 1 and 2 shows the different reactions for epoxydes and genipin agent.

\section{Swelling ratio determination}

The swelling ratio gives a good indication of the hydrophobicity/hydrophilicity of the polymer. This was evaluated by using Eq. (4).

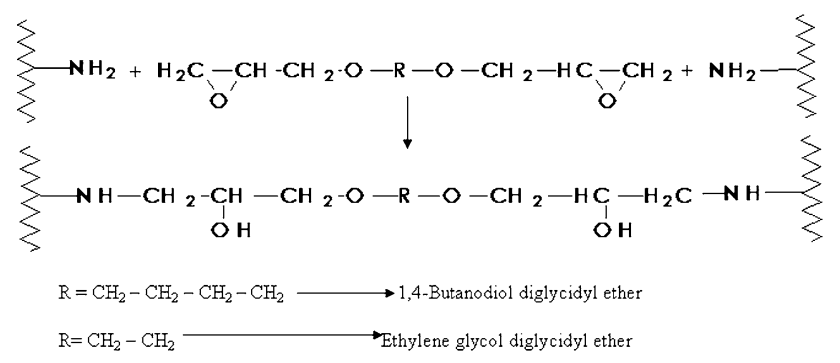

Fig. 1 Crosslinking reaction between gelatine and chitosan and epoxydes
Table 3 Swelling capacities for each gelatine film

\begin{tabular}{lll}
\hline Film & Swelling capacity/\% & Crosslinking agent \\
\hline 1 & 797 & - \\
2 & 696 & CDI at $1 \%$ \\
3 & 608 & CDI at $2 \%$ \\
4 & 645 & CDI at $3 \%$ \\
5 & 356 & Epoxyde 2 at $0.2 \%$ \\
6 & 272 & Epoxyde 2 at $0.5 \%$ \\
7 & 236 & Epoxyde 2 at $1 \%$ \\
8 & 1040 & Genipin at $1 \%$ \\
9 & 967 & Genipin at $2 \%$ \\
\hline
\end{tabular}

The results are indicated in Table 3 for the gelatine films and in Table 4 for the chitosan films.

The results indicated in Table 3 suggest that the swelling capacity:

(1) Is higher when genipin is used as the crosslinkling agent.

(2) Decreases with the increasing crosslinking agent concentration. This could be related to the decreasing of free $-\mathrm{NH} 2$ and $-\mathrm{OH}$ groups of the protein as previously said.

The results indicated in Table 4 show that the swelling capacity of chitosan is lower than the gelatine's. This is related with the lower solubility of the material used and also due to both the epoxydes, although epoxyde 1 presents a slightly higher swelling capacity.

The results suggest that, the higher the degree of crosslinking, the lower the swelling capacity of the films. This result could be related with the fact that, for high levels of crosslinking reaction, exist a greater interaction and linking points between the polymeric chains. So the polymer becomes much more resistant to water absorption. We also could verify that, the films after being crosslinked with the epoxyde 2, presented a lower absorption capacity when compared with the results for the other agents. There is only one exception to these results, and that is the films crosslinked with genipin. The results showed a greater capacity in the films crosslinked that in the gelatine film. This can be explained by the hydrophilicity of the agent.
Fig. 2 Crosslinking reaction between gelatine and genipin<smiles>COC(=O)C1=COC(O)C2C(CO)=CCC12</smiles>

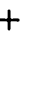
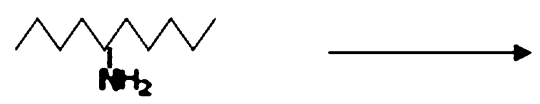

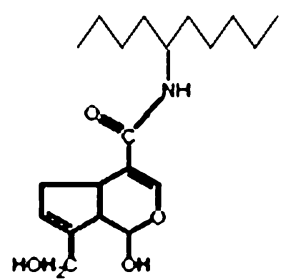


Table 4 Swelling capacities for chitosan films

\begin{tabular}{lll}
\hline Film & Swelling capacity $/ \%$ & Crosslinking agent \\
\hline 10 & 448 & Epoxyde 1 at $1 \%$ \\
11 & 436 & Epoxyde 1 at $2 \%$ \\
12 & 358 & Epoxyde 2 at $0.2 \%$ \\
13 & 291 & Epoxyde 2 at $0.5 \%$ \\
14 & 146 & Epoxyde 2 at $1 \%$ \\
15 & 577 & - \\
\hline
\end{tabular}

Although all the swelling capacities are very high for the purpose of this study, the increase in volume was much greater in length and width than in thickness.

Characterization of the films by using Scanning

Electronic Microscopy (SEM)

The samples $1,2,5,8,10,12$ and 15 were analysed by Scanning Electronic Microscopy (SEM).

From the image of the surface of gelatine, amplified 2000 times, and the transverse cut, amplified 75, 100 and 500 times, we observed a structure in which the threedimensional constitution was a bit collapsed, but with porous sizes between 10 and $100 \mu \mathrm{m}$. We can clearly see a homogeneous structure (Fig. 3).

The surface analysis of film 2 (Gelatine + CDI at 1\%) shows the structure is very similar to the gelatine films without any crosslinking agent. In the transversal cut, amplified 75 and 500 times, we observe a three-dimensional structure forming cocoons with very homogeneous sizes and structures. We also can see a great exposed surface area, which allows a great contact area between the films and the drug included, and the fluid around.

The surface image, amplified 2000 times, of the film 5 (Gelatine + Epoxyde 2 at $0.2 \%$ ), shows a porous surface, with sizes between 5 and $10 \mu \mathrm{m}$. The structures are much smaller and tight than for film 2 .

The images from film 8 (Gelatine + Genipin at 1\%), show a porous surface forming the walls of the bigger porous. In the transversal cut, we can see images similar to

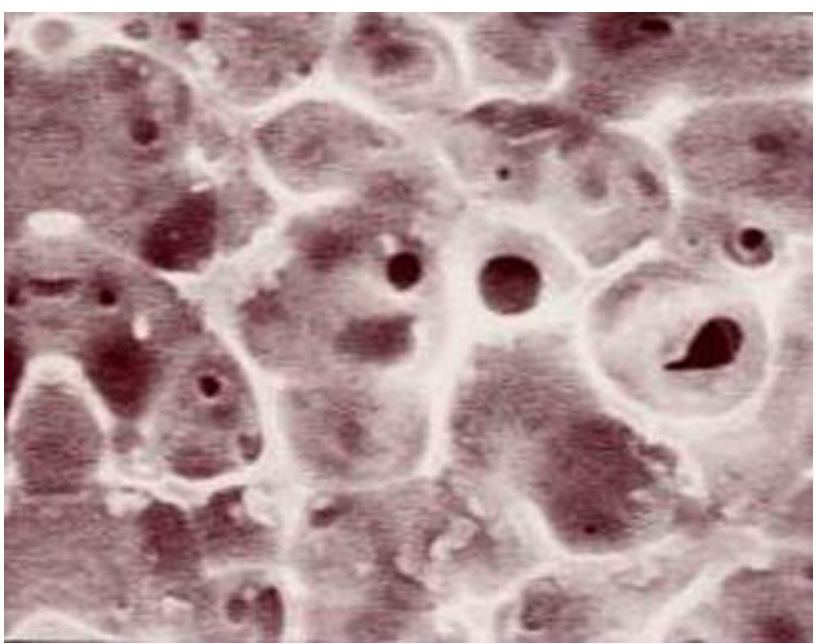

Fig. 4 Surface amplified 3500 times of Chitosan + Epoxyde 1 at $1 \%$

the ones obtained for the gelatine film although there is a greater organization in the gelatine + genipin film.

The chitosan film crosslinked with epoxyde 1 presents porous with constant sizes, approximately $5 \mu \mathrm{m}$ (Fig. 4). But, the other chitosan films studied showed porous with sizes much smaller. This fact can be explained by the structure of chitosan and the crosslinking agents. In fact the chemical structure of epoxyde 2 is much smaller than the one of epoxyde 1 . This fact can explain why the porous of these membranes are smaller.

From all the images, we can suggest that the films obtained presented great porosity. This allows a high contact area, for the linking of the drug, as well as for its release.

\section{Differential Scanning Calorimetry (DSC)}

According to the literature [17], the first glass transition ( Tg) of gelatine occurs around $-30{ }^{\circ} \mathrm{C}$, associated with non-rigid blocks, a second transition between $71^{\circ} \mathrm{C}$ and $75^{\circ} \mathrm{C}$, associated with the rigid blocks of the gelatine, and a third transition around $130{ }^{\circ} \mathrm{C}$, corresponding, probably, to different thermal transitions. The gelatine DSC shows a glass transition around $75.4{ }^{\circ} \mathrm{C}$, visible by the endothermic
Fig. 3 Surface (a) amplified 2000 times and transversal cut (b) amplified 500 times of gelatine film
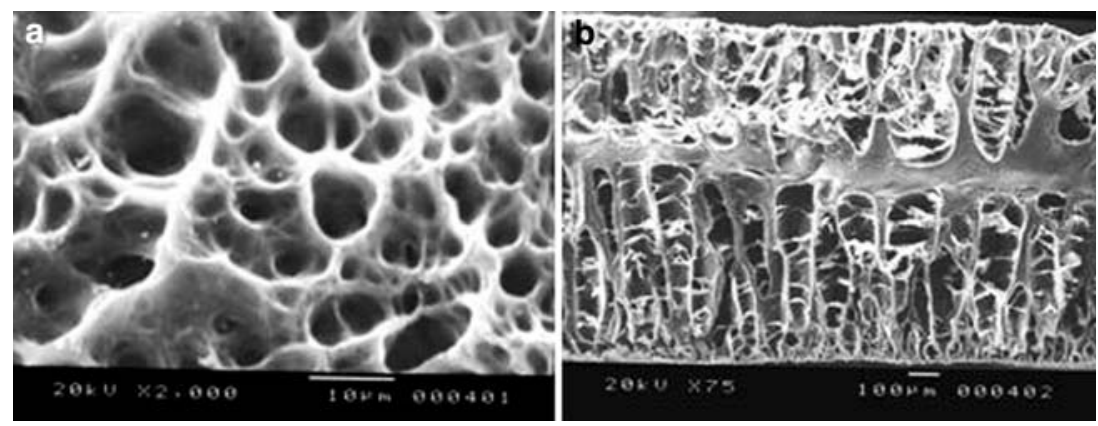


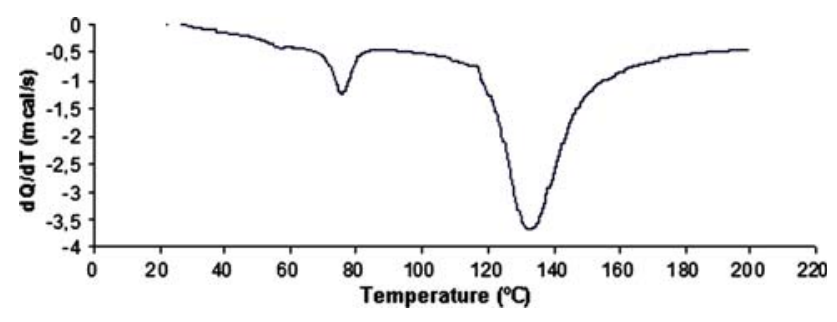

Chart 1 DSC of the gelatine film

depression, caused by the increase of the calorific capacity. Chart 1 shows the DSC obtained for gelatine.

The DSC of film 2 (Chart 2), shows a small increase of the third transition peak, moving from $133{ }^{\circ} \mathrm{C}$ to $141{ }^{\circ} \mathrm{C}$. The peak shows a slight increase of the area, traducing a greater thermal stability. In film 3 , we see a transition at $75{ }^{\circ} \mathrm{C}$ and another at $150{ }^{\circ} \mathrm{C}$. These peaks are less evident and much wide, fact that increases even more when we compare with the DSC of film 4.

The film of gelatine (Chart 3) crosslinked with epoxyde 2 at $0.2 \%$, shows a slight increase of the second transition temperature, from $75.43{ }^{\circ} \mathrm{C}$ to $88.2^{\circ} \mathrm{C}$. We can see a new peak around $160^{\circ} \mathrm{C}$, that can be associated with the decomposition of the crosslinking agent. For film 6 we can't see the peak around $160{ }^{\circ} \mathrm{C}$, which, probably means that the film was not homogenous. The next chart shows the DSC for Gelatine + epoxyde 2 at $0.2 \%$.

Film 8, gelatine crosslinked with genipin (Chart 4), presents an increase of the second transition temperature to $85.0{ }^{\circ} \mathrm{C}$ (Chart 4). The peak around $130{ }^{\circ} \mathrm{C}$ appears in the same position as for gelatine, but it has an area slightly smaller. The peak around $185{ }^{\circ} \mathrm{C}$ is due to the decomposition of the crosslinking agent.

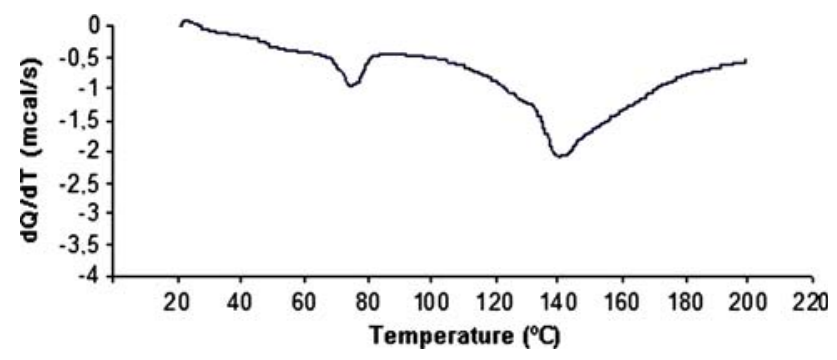

Chart 2 DSC for gelatine crosslinked with CDI at $1 \%$

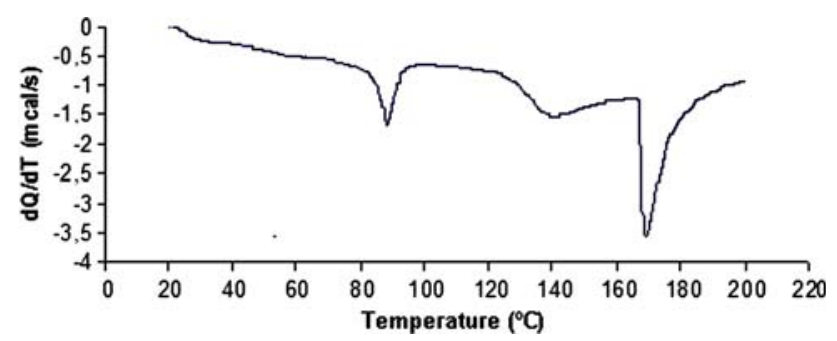

Chart 3 DSC of gelatine crosslinked with epoxyde 2 at $0.2 \%$

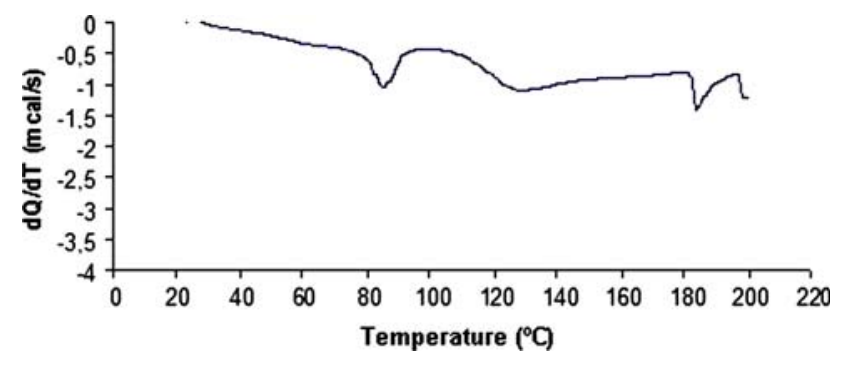

Chart 4 DSC of gelatine + genipin at 1\%

As for the chitosan films, DSC shows a thermal transition around $130{ }^{\circ} \mathrm{C}$ (Chart 5). According to the literature [18] we can see that this transition is normally ascribe to the glass transition temperature, although some authors consider that temperature at $200{ }^{\circ} \mathrm{C}$. The differences obtained are usually related with the molecular weight of the chitosan used. Other transitions also described in the literature were not observed in this study. This can be explained due to the different experimental conditions used in the studies. Those transitions are related with the presence of water in the chitosan chains.

For films 10 and 11, we can see an increase if the second transition temperature, which proves the success of the chemical modification. Furthermore, we can see that film 11, with a greater concentration of crosslinking agent presents a second transition temperature higher than for film 10, which gives the idea that the crosslinking extension is greater for the first film than for the second. Besides this, we can see a decreasing of the fusion enthalpy which can mean a decrease in the polymer crystallinity because we need to supply the sample less energy for the transition to occur.

For films crosslinked with epoxyde 2 (films 12, 13 and 14), we can see also an increase of the second transition temperature. The explanation for this fact is equal for the case of chitosan + epoxyde 1 (films 10 and 11). As for the enthalpy, we can see that the values are equal for these systems which can mean that the crystallinity is equal to chitosan.

As a conclusion, we can see that an increasing of the crosslinking agents concentration, is accomplish with an increase of the thermal transitions and the peaks area. This means that the crosslinking was successful and the chemical reaction was able to stabilize the polymer matrix.

Study of the releasing kinetic of drugs from the polymeric films

The release kinetics were determined with UV spectroscopy at $294.5 \mathrm{~nm}$. After that, we made another test, with a dialysis membrane, to find out the delay caused by the membrane. For that, we put a drug solution inside a dialysis 


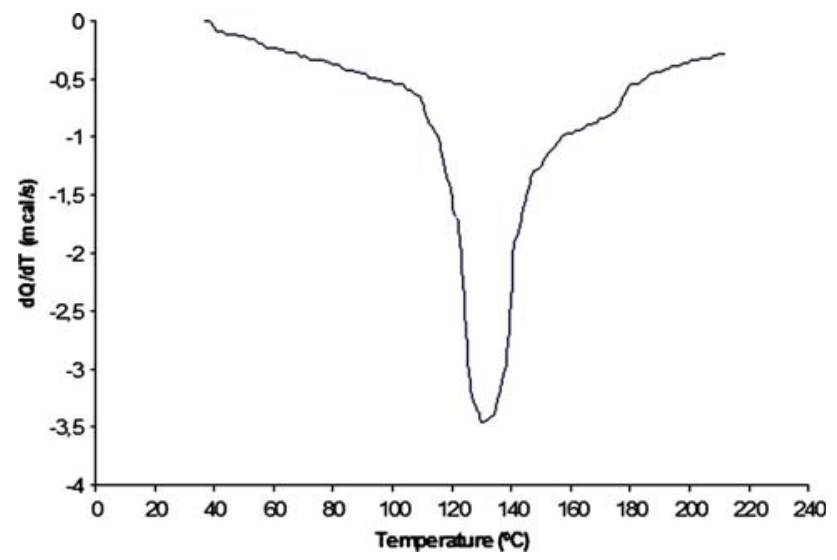

Chart 5 DSC of the chitosan film

membrane and made the readings in the spectrophotometer for different release times. The results suggest that the drug presented a delay of 2 to $3 \mathrm{~h}$, from its release by the system and the passing through the dialysis membrane.

Every film, with a weight between 200 and $260 \mathrm{mg}$ was put inside a dialysis membrane and inside a beaker with serum.

Charts 6 and 7 show the release pattern of films 5 and 12 .

All systems studied showed a similar release pattern and, as can be seen in both charts all systems follow a Fickian kinetic pattern with values for the diffusion release exponent within those considered as Fickian kinetics. As for the diffusion coefficient we applied Eq. (3) to determine it value for each system. The results are shown in Table 5 .

As we can see from the Table 5, the gelatine film without any crosslinking agent is the one that presents a greater diffusion coefficient. This could be related with the open structure that the gelatine molecule presents. When a crosslinking agent is added to the gelatine, the diffusion coefficients begin to get smaller. The same happens with the chitosan systems. The film that presents the biggest

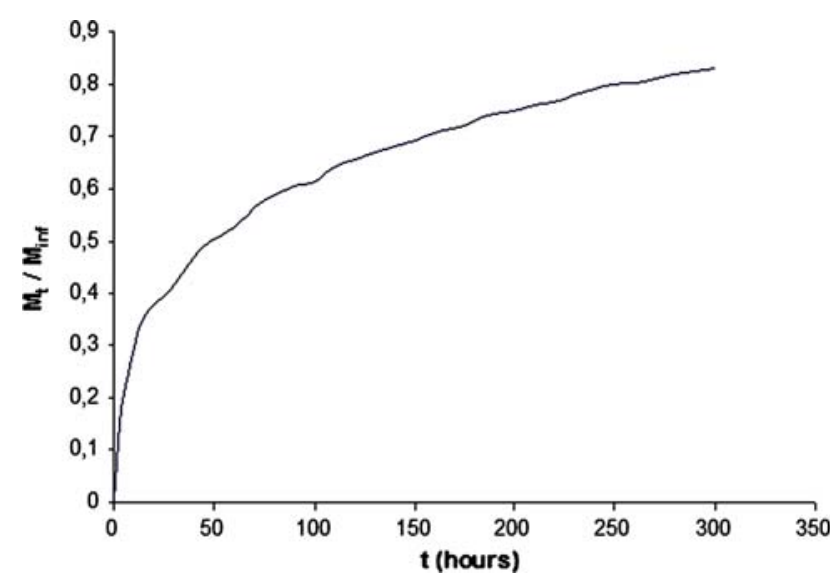

Chart 6 Release pattern of gelatine + epoxyde 2 at $0.2 \%$

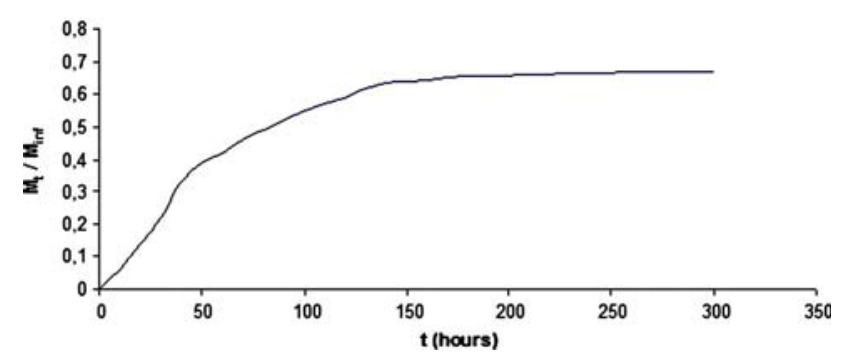

Chart 7 Release pattern for the chitosan and epoxyde 2 at $0.2 \%$ system

Table 5 Diffusion coefficients for Timolol for all the systems studied

\begin{tabular}{ll}
\hline Film & $\begin{array}{l}\text { Diffusion Coefficient } \\
\times 10^{5} /\left(\mathrm{cm}^{2} / \mathrm{h}\right)\end{array}$ \\
\hline 1 & 4.27 \\
2 & 3.18 \\
3 & 3.23 \\
4 & 3.63 \\
5 & 4.09 \\
6 & 3.50 \\
7 & 1.05 \\
8 & 3.28 \\
9 & 2.42 \\
10 & 0.213 \\
11 & 0.154 \\
12 & 0.671 \\
13 & 0.327 \\
14 & 0.308 \\
15 & 1.05 \\
\hline
\end{tabular}

coefficient is the chitosan film, but, as we can see, it's much smaller than the gelatine coefficient. This can be explain due to the fact that the chitosan chains are not so open as the gelatine ones.

We made, just for comparison, a study of the release pattern at $20{ }^{\circ} \mathrm{C}$. The results show that the amount of drug released was much smaller than for the release at $37{ }^{\circ} \mathrm{C}$. This can be related with the degradation of the films at $37^{\circ} \mathrm{C}$. So, we can conclude that the release of the drug, at $37^{\circ} \mathrm{C}$, is not only due to the diffusion of the drug molecules through the polymeric matrix, but also due to the degradation of that matrix.

\section{Biocompatibility and cytotoxicity tests}

As we refer in the materials section, for these tests we used two different types of cells, SRA 01/04 and COS-7. Due to the results presented before, we decided to make these tests only with the gelatine systems. 


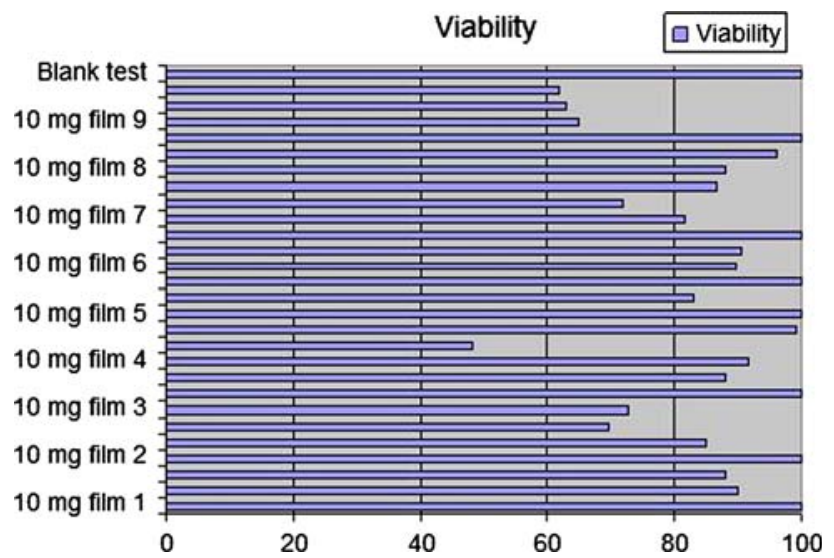

Chart 8 Viability results for the SRA cells

For each test, we made a blank with cells only, without any kind of samples. For these tests we gave $100 \%$ viability and compared the other results with these ones. The results for the SRA cells are shown in Chart 8 .

For each film we made three tests, using first $10 \mathrm{mg}$ of each film, then $30 \mathrm{mg}$ and finally $60 \mathrm{mg}$.

As we can see from the figure, the gelatine film without any crosslinking agent presents high levels of viability. But, for $60 \mathrm{mg}$ of gelatine film we see a decrease in the viability. This can be related to the fact that the levels of $\mathrm{pH}$ dropped in this test, so we can conclude that gelatine, in high amounts make the medium much more acid.

The films of gelatine crosslinked with CDI, present a drop in the levels of viability. But, for lower concentrations, we can still see high levels of viability. It's important to refer at this point that some results may show the contrary to what we are saying but that is due to some lack of homogeneity in the film. That is the case with the results of film 3, where the sample with $30 \mathrm{mg}$ of film presents the higher viability. This problem with the viability is less influent in the case of gelatine crosslinked with epoxyde 2 . For this case, we can see high levels of viability, although the crosslinking agent is toxic. This result was a surprise because, for all the concentrations, we observed high levels of viability, so, we can conclude that all of the epoxyde was linked to the polymeric chain. But, as expected, when the concentration of epoxyde increases, the levels of biocompatibility decreases, but to levels higher than for gelatine crosslinked with CDI.

Finally, for the system Gelatine + Genipin, we observed that the levels of biocompatibility were much smaller than expected, especially when the concentration of genipin increases from $1 \%$ to $2 \%$. The only explanation that we can give is probably many molecules of genipin didn't react and made the system less viable than the others studied.

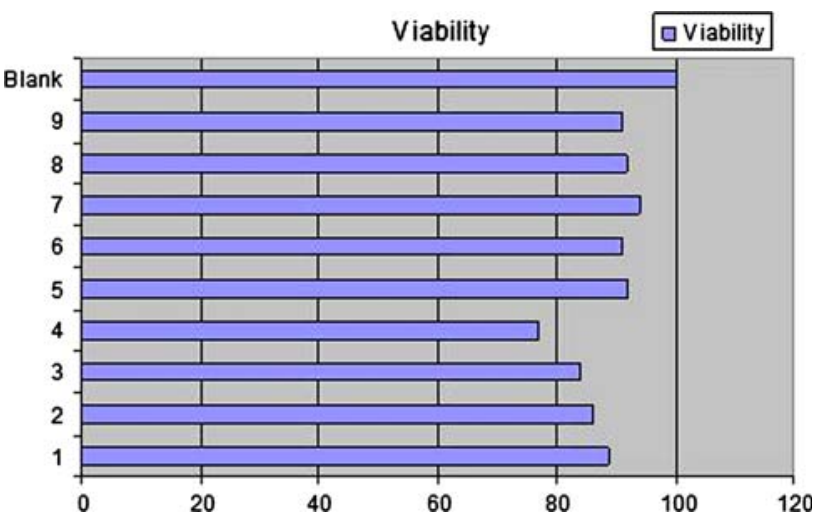

Chart 9 Results of the viability for COS-7 cells

So, we can conclude that cytotoxicity of all the systems is, generally, very low, especially the system gelatine + epoxyde 2 .

As for the COS-7 the method used was the same but with only one amount of each system $(30 \mathrm{mg})$. The results are presented in Chart 9.

As we can see from the figure above, the results show that the viability is, like with the SRA cells, high. Once again the results for the system gelatine + epoxyde are the best ones, even better than for gelatine films. For these cells, the worst result is for the gelatine + CDI system.

Theses results show us that SRA cells are much sensitive than the COS-7, because the viability level for the first cells is much worst than for the COS-7. But, for either type of cells, the levels obtained were very high.

\section{Conclusions}

From this study, we can suggest that, both gelatine and chitosan are good materials for drug delivery systems. We can also say that the method used to modify both materials was good, and the crosslinking was successful. As for the results, we can clearly see that, for all the gelatine systems, the amount of drug released for 7 days was the enough to treat the disease, and also we've observed that the degradation process was well under way at that time. The chitosan systems presented a release pattern much slower to the one we were looking for.

The cytotoxicity of all gelatine systems was very low, especially for the system gelatine + epoxyde 2 , which is the one that presents the best results in all characterizations made. The result with the COS-7 cells gives the option to use these systems in other applications than ophthalmologic ones. 
Acknowledgments The authors would like to thank Fundação para a Ciência e Tecnologia for the financial support to J. F. Almeida (SFRH/BD/17907/2004).

\section{References}

1. Y. WANG et al., Biomaterials 25 (2004) 4279

2. J. T. JACOB, OWEN J. LA COUR and CLAUDE F. BURGOYENE, Biomaterials 22 (2001) 3329

3. R. VAN DER VAKL et al., Ophthalmology 112-7 (2005) 1177

4. M. F. SAETTONE, Future Drug Deliv. Syst. (1998) p. 167

5. M. P. STEVENS, Polymer Chem. (Oxford University Press Inc., London)

6. Y. W. CHIEN, Novel Drug Delivery Systems, Vol. 50 (Dekker, London)

7. J. CRANK, The Mathematics of Diffusion (Clarendon Press, Oxford)
8. S. PUTTIPIPATKHACHRN et al., Journal of Controlled Release 75 (2001) 143

9. V. RISBUD MARKARAND et al., Journal of Controlled Release 68 (2000) 23

10. K. PARK, W. S. W. SHALABY and HAESUN PARK, Biodegradable hydrogels for drug delivery (School of Pharmacy Technomic, Publishing Co, inc, Lancaster Basel)

11. W. E. HENNINK, and C. F. VAN NOSTUM, Adv. Drug Deliv. Rev. 54 (2002) 13

12. F. A. CAREY, Organic Chemistry (The McGraw-Hill Companies, NewYork)

13. A. BIGI et al., Biomaterials 23 (2002) 4827

14. H.- W. SUNG et al., J. Biomed. Mater. Res. 42 (4) (1998) 560

15. R. KONCKI and O. S. WOLFBEIS, Biosens. Bioelectron. 14 (1999) 8792

16. M. H. GIL et al., Biotechnol. Tech. 13 (1999) 595

17. A. N. FRAGA and R. J. J. WILlIAM, Polymer 26 (1985) 113

18. P. FERREIRA et al., J. Carbohydr. Chem. 25 (2006) 233 